\title{
Lineage-specific rapid diagnostic tests can resolve Trypanosoma cruzi Tcll/V/VI ecological and epidemiological associations in the Argentine Chaco
}

Niamh Murphy ${ }^{1 *+}$, Natalia P. Macchiaverna ${ }^{2,3+}$, M. Victoria Cardinal ${ }^{2,3+}$, Tapan Bhattacharyya ${ }^{1}$, Pascal Mertens ${ }^{4}$, Nicolas Zeippen ${ }^{4}$, Yves Gustin ${ }^{4}$, Quentin Gillemann ${ }^{4}$, Ricardo E. Gürtler ${ }^{2,3}$ and Michael A. Miles ${ }^{1}$

\begin{abstract}
Background: Trypanosoma cruzi, the protozoan agent of Chagas disease, is comprised of at least 6 genetic lineages (TCl-TCVI). Their geographical distribution, clinical associations and reservoir hosts are not fully elucidated, as genotyping is hampered due to the difficulty in isolating representative populations of organisms. Lineage-specific serological techniques may address these issues.

Methods: Trypanosoma cruzi lineage-specific serological assays were performed on human, canine, feline and armadillo sera from the Gran Chaco in northern Argentina, a region of ongoing transmission. Synthetic peptides representing lineage-specific epitopes of the trypomastigote small surface antigen (TSSA) were used in ELISA, and the TcIINNVI shared epitope peptide (TSSApep-IINNI) was used in the Chagas Sero K-SeT rapid diagnostic test (RDT).

Results: Chagas Sero K-SeT RDT, using Protein G to detect human and canine lgG, was at least as sensitive as TSSApep-IINNI ELISA using specific secondary antibodies. For sera from humans TSSApep-IINNI seroprevalence by Chagas Sero K-SeT was 273/393 (69.5\%), for dogs 48/73 (65.8\%) and for armadillos 1/7 (14.3\%); by ELISA for cats $5 / 19$ (26.3\%). The seroprevalence for humans was similar to that for Bolivian patients, amongst whom we previously observed an association of TSSApep-IIN/NI seropositivity with severity of cardiomyopathy. In humans, prevalence of TSSApep-IINNI recognition was associated with locality, and with increasing and decreasing age within the Qom and Creole populations, respectively. For dogs TSSApep-IINNI recognition was associated with being born before community-wide insecticide spraying $(P=0.05)$ and with Qom household $(P<0.001)$.

Conclusions: We show here that Chagas Sero K-SeT RDT can replace ELISA for TSSApep-IINNI serology of humans and dogs; for humans there were statistically significant associations between a positive Chagas Sero K-SeT RDT and being resident in Area IV, and for dogs association with Qom household or with being born before the mass spraying campaign; we also show that with cats the TCIINNI epitope can be detected by ELISA. We assessed the lineage distribution in an unprecedented $83 \%$ of the human T. cruzi-seropositive population. These results form the basis for more detailed studies, enabling rapid in-the-field surveillance of the distribution and clustering of these lineages among humans and mammalian reservoirs of T. cruzi infection.
\end{abstract}

Keywords: Trypanosoma cruzi, ELISA, Serology, Lineage-specific, Chagas disease, Argentina, Rapid diagnostic test

\footnotetext{
*Correspondence: niamh.murphy@lshtm.ac.uk

${ }^{\dagger}$ Niamh Murphy, Natalia P. Macchiaverna and M. Victoria Cardinal

contributed equally to this work

${ }^{1}$ Faculty of Infectious \& Tropical Diseases, London School of Hygiene \&

Tropical Medicine, London, UK

Full list of author information is available at the end of the article
}

(c) The Author(s) 2019. This article is distributed under the terms of the Creative Commons Attribution 4.0 International License (http://creativecommons.org/licenses/by/4.0/), which permits unrestricted use, distribution, and reproduction in any medium, provided you give appropriate credit to the original author(s) and the source, provide a link to the Creative Commons license, and indicate if changes were made. The Creative Commons Public Domain Dedication waiver (http://creativecommons.org/ publicdomain/zero/1.0/) applies to the data made available in this article, unless otherwise stated. 


\section{Background}

Chagas disease, caused by infection with the protozoan parasite Trypanosoma cruzi, remains a major public health problem in endemic regions of Latin America. The initial acute phase of infection may be asymptomatic or have mild and non-specific symptoms but can be fatal, particularly in infants, young adults or the immunocompromised. Without successful treatment $T$. cruzi infection is life-long: the immune response reduces the level of infection but is unable to eliminate it, as is apparent from xenodiagnosis or PCR of seropositive patients, and recrudescent parasitaemia in the immunocompromised. In the chronic phase, around $30 \%$ of those infected will develop chagasic heart disease, and a proportion will also have gastrointestinal megasyndromes [1, 2]. Of the 1.5 million people in Argentina infected with T. cruzi, approximately 370,000 are estimated to have chagasic cardiomyopathy [3].

Ongoing transmission is primarily maintained by contamination with T. cruzi infected faeces of the predominant local triatomine insect vector, Triatoma infestans, which infests rural dwellings, especially in the Gran Chaco region, where vector control has had limited success [4]. Transmission can also be oral by consumption of triatomine faeces-contaminated food or congenitally, and via T. cruzi infected blood or organ donors. Trypanosoma cruzi infection is a zoonosis: dogs, cats and rodents associated with households are reservoir hosts, with evidence of a positive association between the number of infected dogs and the prevalence of human infection [5]. A wide range of sylvatic mammals carry $T$. cruzi infection [6].

Trypanosoma cruzi is currently understood to comprise six genetic lineages TcI-TcVI [7], with TcBat proposed as a seventh lineage, related to TcI [8]. Based on genotyping, TcII/V/VI lineages predominate in the domestic cycle in southern cone countries, including Argentina. However, genotyping may be biased by nonrepresentative isolation of $T$. cruzi, which has sequestered intracellular replication and only scanty chronic blood infections, and by competitive selection in vitro between the lineages.

The polymorphic trypomastigote small surface antigen (TSSA), expressed on bloodstream trypomastigotes, has been the only antigen applicable for indirect, serological identification of lineage(s) carried by a patient or reservoir host [9]. TcI, TcIII and TcIV each have their own distinct potential TSSA epitope. At the same site a distinct amino acid sequence is shared by TcII/V/VI, and the hybrids TcV/VI also have a second sequence, as they are heterozygous and have two haplotypes at that locus [10]. Recombinant TSSA produced in E. coli or synthetic peptide epitopes (TSSApep) have been used with Argentine chagasic samples for T. cruzi lineage-specific serology [9, 11-21], particularly with the isoform common to TcII/V/ VI; the recombinant form has also been used for canine serology [12, 22].

We recently developed the novel rapid diagnostic test (RDT) Chagas Sero K-SeT incorporating TSSApep-II/V/ $\mathrm{VI}$ and found that response to this RDT was associated with severity of cardiomyopathy in Bolivian patients [23]. As Chagas Sero K-SeT uses Protein G to detect IgG, this same test should be directly applicable to both humans and diverse mammal species.

Here, our objectives were to apply T. cruzi lineage-specific TSSApep ELISA and the Chagas Sero $K$-SeT RDT to humans and mammals of the Chaco region of northern Argentina to gain further insight on ecological and epidemiological associations, focusing here on TcII/V/VI.

\section{Methods}

Serum samples from seropositive patients and T. cruziinfected animals were from archives stored at the University of Buenos Aires.

\section{Study sites}

The two study sites were the municipalities of Pampa del Indio and Avia Terai in Chaco Province, northern Argentina. The majority of samples tested were from a larger ongoing project on the eco-epidemiology and control of Chagas disease, taking place in the rural area of Pampa del Indio $\left(1600 \mathrm{~km}^{2}\right)$, consisting of 1446 inhabited households in 30 villages [24]. There are two main ethnic groups inhabiting the area, Creole and Qom; the latter make up half of the local population, but are unevenly distributed among the rural villages [25]. For logistic reasons we divided the rural area into 4 study areas (named Areas I-IV). Vector control activities included a baseline house infestation assessment, followed by a communitywide spraying with pyrethroid insecticides, which took place between 2007-2009, complemented by periodic entomological surveys and community-based surveillance to detect re-infestation [5, 26-28].

In 2015, research activities were expanded to include Avia Terai municipality $\left(770 \mathrm{~km}^{2}\right)$, around $150 \mathrm{~km}$ from Pampa del Indio. This municipality comprises 307 rural households, inhabited by a Creole population. Figure 1 shows typical dwellings and environment of the study sites.

\section{Sample collection Human samples}

Trypanosoma cruzi seropositive human samples were obtained in different serosurveys that took place from August 2014 until July 2017. Serum samples were examined using conventional serology by means of two ELISAs 

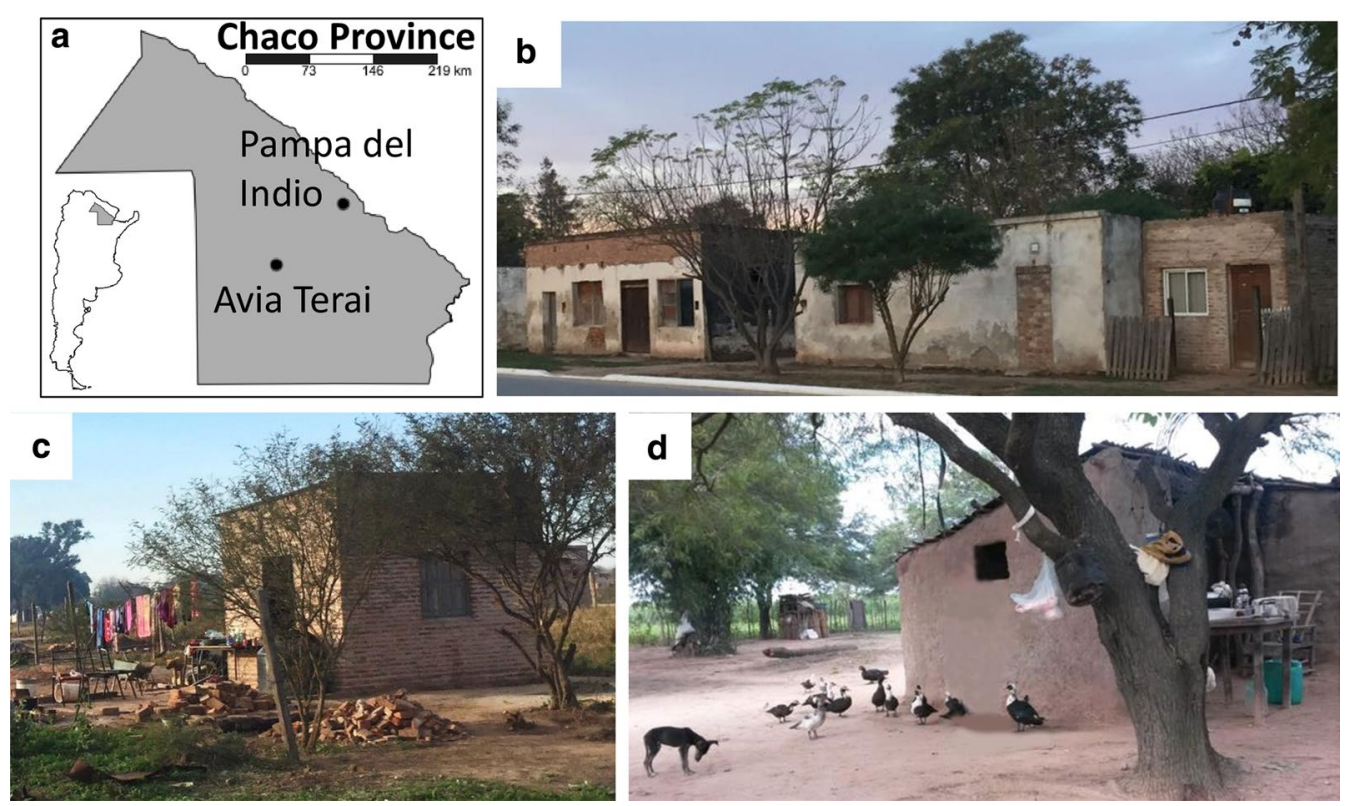

Fig. 1 Study setting. a Location of Pampa del Indio and Avia Terai study sites in Chaco Province, Argentina. b Typical periurban dwelling. c, d Typical rural dwelling and environment in Avia Terai (c) and Pampa del Indio (d)

using either semipurified fractions of epimastigote lysate (Chagatest, Wiener lab, Argentina) or recombinant antigens (ELISA Rec V3.0, Wiener lab). A patient was considered Chagas seropositive if reactive in both tests. Serologically discordant samples were tested by an indirect immunofluorescence antibody test (IFAT) (Iffluor Parasitest Chagas, Laboratorio IFI, Buenos Aires, Argentina) or submitted to the reference diagnosis laboratory at the National Institute of Parasitology "Dr. Mario Fatala Chabén" (Buenos Aires, Argentina) for a final diagnosis. In addition, 10 T. cruzi seronegative human samples from Buenos Aires (a non-endemic area) presenting with other pathologies and 20 seronegative samples from the study sites were assayed by Chagas Sero $K$-SeT.

\section{Animal samples}

In 2008, cross sectional house-to-house surveys were carried out targeting all dogs and cats within 7 contiguous villages of Pampa del Indio considered to have a high infestation of T. cruzi infected triatomine bugs. Owners were interviewed via questionnaire and asked for further information on whether they had permanent residence in the study village or came from other villages outside the study area [25]. Additional samples were collected during a dog survey carried out in June 2016 (Cardinal et al., unpublished). Dogs and cats $\geq 4$ months of age were examined by serology and younger animals and cats were examined by xenodiagnosis. Up to $7 \mathrm{ml}$ of blood were taken from the animals by trained and experienced field personnel, and processed and stored as previously described [29]. A dog or cat was considered infected with $T$. cruzi if it was seroreactive with at least two serological tests (i.e. seropositive by ELISA and indirect haemagglutination test) or if it was xenodiagnosis-positive.

Trypanosoma cruzi-infected armadillos were captured using traps baited with beef or chicken strips soaked in fish sauce in different trapping surveys from August 2008 to August 2011. Traps were checked every morning and re-baited when needed. Full capture and sampling methods are described elsewhere [30]. Armadillos were examined for infection by xenodiagnosis as described [31] and not by conventional serology.

\section{TSSA lineage-specific serology}

A total of 393 human, 85 dog (Canis familiaris), 19 cat (Felis catus) and 7 armadillo (6 Dasypus novemcinctus and 1 Tolypeutes malacus) serum samples were tested here by TSSApep lineage-specific ELISA and/or the Chagas Sero $K$-SeT RDT. A subset of 38/393 human and 73/85 dog serum samples were tested by both TSSApepII/V/VI ELISA and Chagas Sero K-SeT. All these human and dog samples tested by both lineage-specific serology methods were positive by conventional serology.

\section{TSSApep lineage-specific ELISA}

ELISAs were performed with synthetic peptides TSSApep-II/V/VI, -III, -IV and -V/VI representing residues $37-52$ in the TSSA protein of those lineages (Additional 
file 1: Table S1) and with a control reference T. cruzi TcII lysate (IINF/PY/00/Chaco23) as described previously [17], with the modifications described below for human, canine and feline samples. In all cases, two replica plates were run simultaneously. Cut-offs were determined by first subtracting the plate background (no antigen wells) absorbance values from the mean reading for each sample; those samples that were then greater than five standard deviations higher than seronegative controls were considered positive.

\section{Human samples}

This was performed as described previously [17], with the following modifications: $0.1 \mu \mathrm{g}$ of each TSSApep was used per well; goat anti-human IgG-HRP (074-1006: SeraCare, USA) diluted 1:5000 was used; reaction wells were developed with $100 \mu \mathrm{l}$ of ABTS substrate (50-6200: SeraCare) and stopped with $50 \mu \mathrm{l}$ of stop solution; absorbance values were determined at a wavelength of $405 \mathrm{~nm}$.

\section{Dog and cat samples}

ELISA plates were coated directly with each TSSApep at $0.1 \mu \mathrm{g} / 100 \mu \mathrm{l} /$ well in coating buffer overnight. After blocking and washing steps as described [17], $100 \mu \mathrm{l}$ of 1:200 (dog) or 1:500 (cat) dilutions of sera were applied. Subsequently, $100 \mu \mathrm{l}$ of goat anti-dog IgG-HRP (14-1906 , SeraCare) diluted 1:12,000, or goat anti-cat IgG-HRP (14-20-06, SeraCare) diluted 1:5000, was used, prior to addition of substrate.

\section{Chagas Sero $K$-SeT RDT}

This novel RDT, manufactured at Coris BioConcept, employed TSSApep-II/V/VI as the antigen and Protein $\mathrm{G}$ as the detection molecule for IgG, as previously described [23]. Tests were visually assessed at $15 \mathrm{~min}$ maximum incubation time and considered valid if the control band was present; the additional presence of a test line band of any intensity was considered positive for TSSApep-II/V/VI recognition. The absence of test line band was considered a negative test. The presence of the test band was determined by visual inspection of the RDT, independently by two individuals.

\section{Trypanosoma cruzi genotyping}

Trypanosoma cruzi lineage was determined by PCR of the genomic targets spliced-leader (SL) DNA, 24S $\alpha$ ribosomal RNA genes and A10 from T. cruzi isolates [32, 33]. For humans only, a second PCR-based protocol targeting two nuclear genes (TcSC5D and TcMK) [34] was also employed [35] to allow for classification of lineages TcITcVI as well as TcBat and TcV/VI [34].

\section{Statistical analyses}

Fisher's exact test (two tailed) was used to calculate odds ratios, 95\% confidence intervals and $P$-values (StataCorp. 2019. Stata Statistical Software: Release 15. StataCorp LLC, Texas, USA). A $P$-value $\leq 0.05$ was considered significant. A Kappa test was used to determine the level of agreement between the TSSApep-II/V/VI ELISAs and Chagas Sero $K$-SeT RDT, the degree of agreement was qualified by Kappa and categorized as mild, moderate or severe and 95\% confidence intervals calculated (GraphPad, San Digeo, USA). For seropositive humans from Area II and IV, we performed univariate and multivariate (generalized linear model, GLMs) analyses to detect factors associated with RDT reactivity by means of a logistic regression. The full model tested was: RDT reactivity $\sim$ age at diagnosis $v s$ ethnic group + study area + gender + occurrence of $T$. infestans in the household + another cohabitant with reactive RDT. Linear regressions were calculated for each ethnic group. For Creoles, we forced the origin in $100 \%$. Univariate analysis of dog RDT reactivity was performed for animals examined for diagnosis in 2008.

\section{Results}

A total of 373 human, 85 dog and 19 cat samples were seropositive as described in Methods. Seven armadillos were positive by xenodiagnosis. Additionally, 20 human samples were seronegative by conventional serology. Most $(292 / 393,74.3 \%)$ of the human samples belonged to 10 rural villages in Area II and Area IV from Pampa del Indio, where we aimed at full coverage of the detected seropositive population. In these villages a total of 1338 inhabitants were serodiagnosed and 332 (24.8\%) found seropositive for T. cruzi (Macchiaverna et al., unpublished) with $88.0 \%(292 / 332)$ of these seropositive patients assayed by Chagas Sero $K$-SeT RDT.

\section{Chagas Sero $K$-SeT is more sensitive than TSSApep-II/V/VI ELISA for humans and dogs}

Comparing the TSSApep-II/V/VI ELISA and the Chagas Sero $K$-SeT RDT, all human samples that were positive by TSSApep-II/V/VI ELISA were also positive by Chagas Sero $K$-SeT RDT for recognition of this peptide; Figure 2 shows examples of correspondence between these methods. However, this RDT additionally identified 10 human samples as positive that were negative by TSSApep-II/V/ VI ELISA (Table 1), although seropositive by conventional serology. Thus, for human samples tested by both methods, $13 / 38$ (34\%) were TSSApep-II/V/VI ELISA positive whereas $23 / 38$ (61\%) were positive by Chagas Sero $K$-SeT. Consequently the Kappa statistic showed moderate agreement between the two tests $(0.51 ; 95 \% \mathrm{CI}$ : 


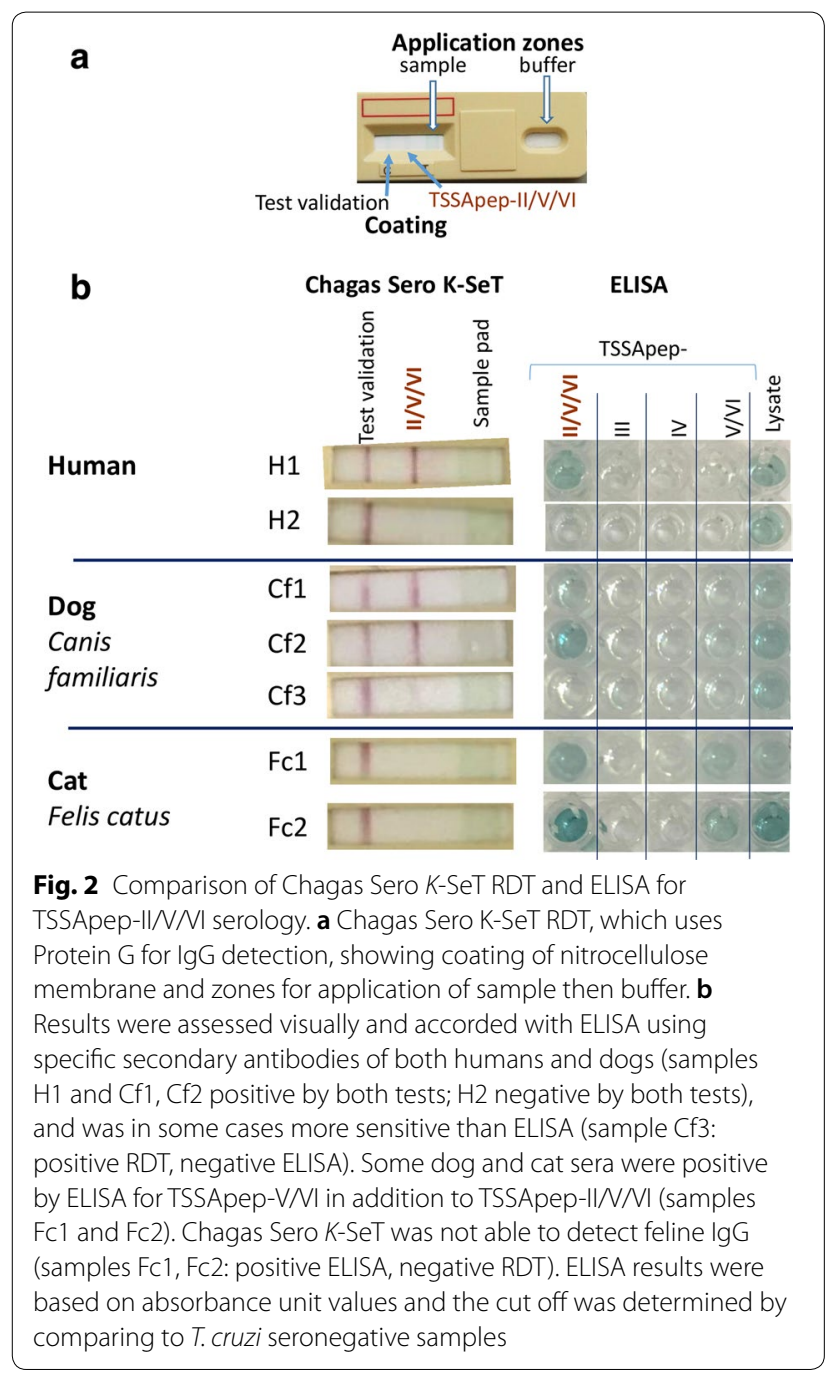

0.28-0.74). Similarly, all dog samples that were positive by TSSApep-II/V/VI ELISA were also positive by Chagas Sero $K$-SeT RDT (Fig. 2); among these samples tested by both methods, 33/73 (45\%) were TSSApep-II/V/VI ELISA positive whereas an additional 15 were positive by Chagas Sero $K$-SeT only (48/73; 66\%). Here, the Kappa statistic found a good agreement between the two tests
(0.60; 95\% CI: 0.44-0.77). Furthermore, the Protein G conjugate in Chagas Sero $K$-SeT was highly effective in detecting binding of both human and canine IgG to TSSApep-II/V/VI, without the need for the specific secondary antibodies used in the ELISA.

\section{TSSApep ELISA}

Table 1 shows the TSSApep lineage-specific ELISA results for human, dog and cat samples. For humans, ELISA reaction to TSSApep-II/V/VI occurred with $13 / 38(34.2 \%)$ sera, whereas it was $33 / 85$ (38.8\%) for dog and $5 / 19(26.3 \%)$ for cat. Interestingly, of these animal samples $12 \mathrm{dog}$ and all 5 cat samples were additionally positive for TSSApep-V/VI, which differs from TSSApepII/V/VI by a single amino acid substitution (Additional file 1: Table S1).

\section{Chagas Sero K-SeT RDT}

In total across the two study sites the detected prevalences of TcII/V/VI infection by Chagas Sero $K$-SeT in humans $(273 / 393,69.5 \%)$ and dogs $(48 / 73,65.8 \%)$ were similar (Table 1). However, as expected due to the lack of Protein G efficacy with cats, Chagas Sero $K$-SeT was negative with sera of $2 / 2$ cats strongly seropositive for TSSApep-II/V/VI by ELISA (Fig. 2). For armadillos, 1/7 $(14.3 \%)$ was weakly positive with Chagas Sero $K$-SeT (Fig. 2).

\section{Comparison of lineage-specific serology with genotyping}

Corresponding $T$. cruzi genotyping data were available for a subset of the human, dog, cat and armadillo samples tested by TSSApep lineage-specific serology (Additional file 1: Table S2). A total of 28 of 38 human serum samples with genotyping data were reactive by Chagas Sero $K$-SeT. Given that all human-infecting lineages were genotyped as $\mathrm{TcV}$ or TcVI, the sensitivity of Chagas Sero $K$-SeT was $73.7 \%$ (95\% CI: $57.8-85.1 \%$ ). None of the 10 T. cruzi seronegative serum samples from non-endemic patients with other pathologies was reactive by Chagas Sero $K$-SeT. However, 8 of 20 sera from the study sites that were negative with our conventional serology were reactive by Chagas Sero $K$-SeT. Overall, 8/30 samples

Table 1 Human, dog and cat samples assayed by TSSApep ELISA and/or Chagas Sero K-SeT RDT

\begin{tabular}{|c|c|c|c|c|c|c|c|c|c|}
\hline \multirow[t]{2}{*}{ Host } & \multicolumn{4}{|c|}{ Assayed by both ELISA and RDT } & \multicolumn{5}{|c|}{ Individual assay positives } \\
\hline & $\begin{array}{l}\text { RDT and } \\
\text { ELISA positive }\end{array}$ & RDT only positive & $\begin{array}{l}\text { ELISA only } \\
\text { positive }\end{array}$ & $\begin{array}{l}\text { RDT and ELISA } \\
\text { negative }\end{array}$ & IIN/VI ELISA & III ELISA & IV ELISA & VNI ELISA & RDT \\
\hline Human & $13 / 38$ & $10 / 38$ & 0 & $15 / 38$ & $13 / 38$ & 0 & 0 & 0 & $273 / 393(69.5 \%)$ \\
\hline Dog & $33 / 73$ & $15 / 73$ & 0 & $25 / 73$ & $33 / 85$ & 0 & 0 & $12 / 85^{a}$ & $48 / 73(65.8 \%)$ \\
\hline Cat & 0 & 0 & $2 / 2$ & 0 & $5 / 19$ & 0 & 0 & $5 / 19^{a}$ & $0 / 2$ \\
\hline
\end{tabular}

a Also positive by TSSApep-II/V/VI ELISA 
Chagas seronegative by conventional serology were reactive by the RDT, thus estimated specificity was $73.3 \%$ (95\% CI: $55.5-86.0 \%)$.

Of the 17 dogs for which the T. cruzi genotype was TcII/V/VI or TcVI, 11/17 were positive for TSSApepII/V/VI by Chagas Sero K-SeT (7 were TSSApep-II/V/VI ELISA positive only, 4 were additionally TSSApep-V/VI positive, and 6 were TSSApep ELISA negative); $2 / 17$ were negative by both lineage-specific serological methods. For the single dog from which TcIII was genotyped, the corresponding serum was Chagas Sero $K$-SeT positive but TSSApep ELISA negative.

Of the four cats for which T. cruzi was genotyped as TcII/V/VI or TcVI, all were negative by TSSApep ELISA. The single armadillo that was TSSApep-II/V/VI positive by Chagas Sero K-SeT had T. cruzi genotyped as TcIII, as were the remaining armadillos for which these genotyping data were available.

\section{Hosts, clustering and ecological associations}

Among the two sites (Pampa del Indio and Avia Terai), in Pampa del Indio 242/350 (69.1\%) were positive by Chagas Sero $K$-SeT compared to 31/43 (72.1\%) in Avia Terai, but this was not statistically significant (OR: 0.8 ; $95 \% \mathrm{CI}$ : $0.4-1.7 ; P=0.69$ ).

Univariate associations of TSSA-II/V/VI seropositivity by Chagas Sero $K$-SeT within the Pampa del Indio study Areas II and IV (humans and dogs) are shown in Table 2. For humans, there were no significant associations between TSSApep-II/V/VI recognition and age, ethnicity, previously infested house, gender or having another householder TSSA-II/V/VI positive. A significantly higher Chagas Sero $K$-SeT reactivity was observed for patients inhabiting Area IV compared to Area II (OR: 2.07; 95\% CI: $1.15-3.88 ; P=0.02$ ).

For dogs born before the mass insecticide spraying programme there was a significant association with TSSApep-II/V/VI seropositivity (OR: 8.70; 95\% CI: 0.78436.49; $P=0.046)$. Interestingly, unlike for humans, Qom household was also significantly associated with TSSApep-II/V/VI recognition in dogs (OR: 8.39; 95\% CI: 1.7378.91; $P=0.003$ ). We found no evidence of significant associations between TSSApep-II/V/VI recognition and the roles and behaviour of dogs, such as sleeping inside or hunting (Table 2).

For the 19 cats that were assessed according to the available information, there were no significant associations between recognition of TSSApep-II/V/VI or TSSApep-V/VI, Qom or Creole ownership, hunting, domestication and sleeping habits (data not shown).

By means of multivariate regression analysis variables associated with Chagas Sero $K$-SeT seropositivity were identified. A significant interaction between age and ethnicity was observed: for Qom the reactivity increased with age, whilst for Creoles it decreased (Table 3 and Fig. 3). For Creoles, the percentage of RDT reactive persons decreased with age with a significant slope of $-0.72 *$ age (in years) $\left(R^{2}=70.34, P=0.0003\right)$ whereas for Qom the reactivity increased with a slope of $0.25 *$ age, though it was marginally significant $\left(R^{2}=45.27\right.$, $P=0.098)$. As observed in the univariate analysis, inhabitants from Area IV exhibited a higher reactivity than those from Area II. No significant associations were observed with the other variables evaluated (Table 3).

\section{Discussion}

We have previously applied TSSApep lineage-specific ELISA to human chagasic sera [17] and to sylvatic primate hosts of T. cruzi [36], and adapted TSSApep-II/V/ VI serology to the Chagas Sero K-SeT RDT [23]. Here, we deployed TSSApep serology as a rapid and efficient means for surveillance of $T$. cruzi lineage distribution among humans and animals in active transmission cycles in the Chaco region of northern Argentina.

Previous reports using TSSA serology on Argentine chagasic samples have been principally based on ELISAs and immunoblotting [9, 11-16, 18-21]. Here, we applied TSSApep-II/V/VI serology in a user-friendly, low cost RDT format, applicable at point-of-care to patients. We show excellent concordance between the performance of the Chagas Sero $K$-SeT and TSSApep-II/V/VI ELISAs in humans, as also seen with Bolivian sera [23]. However, more samples tested by both lineage-specific methods were positive with the RDT, suggesting potentially either a greater sensitivity or lower specificity. However, in silico analysis and sequencing of the TSSA gene from T. cruzi encompassing a range of hosts and geographical locations has not identified any novel epitopes (unpublished observations). Furthermore, none of the 30 seronegative samples was positive by this RDT, indicating that the RDT has greater sensitivity, as might be expected because the RDTs employ higher serum concentrations. Using the observed sensitivity and specificity we estimated that the prevalence of infection with TcII/V/VI in the seropositive human population of Pampa del Indio is $88.2 \%$ (95\% CI: 76.4-99.0\%), which provides further support for the prevalence of hybrid lineages in infected humans from the Chaco, as indicated by time-consuming artificial xenodiagnosis, in vitro culture, parasite isolation and PCR-based lineage identification [35].

Interestingly, the prevalence of Chagas Sero $K$-SeT RDT positives in these Argentine patients $(69.5 \%$ for Pampa de lndio and Avia Terai combined) is similar to that seen in Bolivian patients (66.9\%) amongst whom we observed an association with severity of cardiomyopathy [23]. Moreover, the significant differences observed in the 
Table 2 Univariate analyses of hosts, clustering and ecological associations with Chagas Sero K-SeT (Pampa del Indio)

\begin{tabular}{|c|c|c|c|c|c|}
\hline & Category & $n$ & No. positive (\%) & OR $(95 \% \mathrm{Cl})$ & $P$-value \\
\hline \multirow[t]{16}{*}{ Humans } & Age & 292 & $199(68.2)$ & $1.00(0.98-1.01)$ & 0.62 \\
\hline & \multicolumn{5}{|l|}{ Ethnicity } \\
\hline & Creole & 68 & $50(73.5)$ & 1 & \\
\hline & Qom & 224 & $149(66.5)$ & $0.72(0.38-1.29)$ & 0.28 \\
\hline & \multicolumn{5}{|l|}{ Gender } \\
\hline & Female & 144 & $100(69.4)$ & 1 & \\
\hline & Male & 148 & $99(66.9)$ & $0.89(0.54-1.46)$ & 0.64 \\
\hline & \multicolumn{5}{|l|}{ Study area } \\
\hline & $\|$ & 212 & $136(64.2)$ & 1 & \\
\hline & IV & 80 & $63(78.8)$ & $2.07(1.15-3.88)$ & $0.02^{*}$ \\
\hline & \multicolumn{5}{|c|}{ Presence of T. infestans in the household } \\
\hline & No & 59 & $43(72.9)$ & 1 & \\
\hline & Yes & 233 & $156(67)$ & $0.75(0.39-1.40)$ & 0.38 \\
\hline & \multicolumn{5}{|c|}{ Cohabitant with reactive RDT } \\
\hline & No & 146 & $102(69.9)$ & 1 & \\
\hline & Yes & 146 & $97(66.4)$ & $0.85(0.52-1.40)$ & 0.53 \\
\hline \multirow[t]{18}{*}{ Dogs } & \multicolumn{5}{|l|}{ Function } \\
\hline & Guardian & 41 & $26(63.4)$ & 1 & \\
\hline & Hunting & 32 & $23(71.9)$ & $1.47(0.49-4.59)$ & 0.47 \\
\hline & \multicolumn{5}{|l|}{ Type of hunting } \\
\hline & Sylvatic animals & 34 & $23(67.6)$ & 1 & \\
\hline & Not hunting & 16 & $11(68.8)$ & $1.05(0.25-4.84)$ & 1.00 \\
\hline & \multicolumn{5}{|l|}{ Place of birth } \\
\hline & In study area & 52 & $36(69.2)$ & 1 & \\
\hline & Not in study area & 12 & $9(75)$ & $1.33(0.28-8.63)$ & 1.00 \\
\hline & \multicolumn{5}{|l|}{ Ethnicity of the household } \\
\hline & Creole & 57 & $31(54.4)$ & 1 & \\
\hline & Qom & 22 & $20(90.1)$ & $8.39(1.73-78.92)$ & $0.00^{*}$ \\
\hline & \multicolumn{5}{|l|}{ Born after mass spraying } \\
\hline & Yes & 5 & $1(20)$ & 1 & \\
\hline & No & 73 & $50(68.5)$ & $8.70(0.78-436.49)$ & $0.05^{*}$ \\
\hline & \multicolumn{5}{|l|}{ Place of sleeping } \\
\hline & Outside of the domicile & 18 & $12(66.7)$ & 1 & \\
\hline & Inside of the domicile & 14 & $12(85.7)$ & $3.00(0.41-35.07)$ & 0.41 \\
\hline
\end{tabular}

Note: Areas II and IV only. In each case, only those samples where the examined information was known were included in the analysis

* Statistical significance $(P \leq 0.05)$

Abbreviations: $\mathrm{OR}$, odds ratio; $\mathrm{Cl}$, confidence interval

Chagas Sero $K$-SeT RDT reactivity between study Areas II and IV of Pampa del Indio and the different trends for age in the two ethnic groups merits further study, given that this may be related to different incidence rates of cardiomyopathy.

One strength of this study is the level of coverage of the seropositive human population achieved. Overall, considering Area II and IV from Pampa del Indio, 59.9\% of seropositive inhabitants yielded T. cruzi lineage identification. There is no precedent in the literature of such coverage in a well-defined human population. Most of the previous lineage identification studies comprised human samples collected in hospitals; therefore, the geographical or epidemiological context where the infection originated remains unclear [37-44]. Another constraint for mass lineage identification is the complexity of the traditional genotyping methods, which usually require $T$. cruzi isolation or large blood samples.

There was no association between ethnic group and overall human seroprevalence in Areas II and IV of Pampa del Indio. Nevertheless, Qom communities in Area III are predicted to have higher seroprevalence than 
Table 3 Multivariate analyses for associations with Chagas Sero K-SeT, Areas II and IV, Pampa del Indio

\begin{tabular}{llll}
\hline Category & OR $(95 \% \mathrm{Cl})$ & $P$-value & $\mathrm{Rl}$ \\
\hline Age & $0.98(0.93-1.02)$ & 0.31 & 0.48 \\
Ethnicity & & & 0.45 \\
$\quad$ Creole & 1 & & \\
Qom & $0.38(0.05-3.06)$ & 0.36 & \\
Gender & & & 0.27 \\
$\quad$ Female & 1 & & \\
Male & $0.93(0.56-1.55)$ & 0.78 & \\
Area & & & $0.82^{*}$ \\
॥ & 1 & & \\
IV & $2.05(1.08-3.88)$ & $0.03^{*}$ & \\
Presence of T. infestans in the dwelling & & & 0.41 \\
$\quad$ No & 1 & & \\
Yes & $0.69(0.36-1.32)$ & 0.26 & \\
Cohabitant with reactive RDT & & & 0.32 \\
$\quad$ No & 1 & & \\
Yes & $0.82(0.49-1.38)$ & 0.45 & \\
Age vs ethnicity & & & 0.23 \\
Age vs Creole & 1 & & \\
Age vs Qom & $1.04(1.00-1.09)$ & $0.04^{*}$ & \\
A & & &
\end{tabular}

Abbreviations: $\mathrm{OR}$, odds ratio; $\mathrm{Cl}$, confidence interval; $\mathrm{RI}$, relative importance * Statistically significant

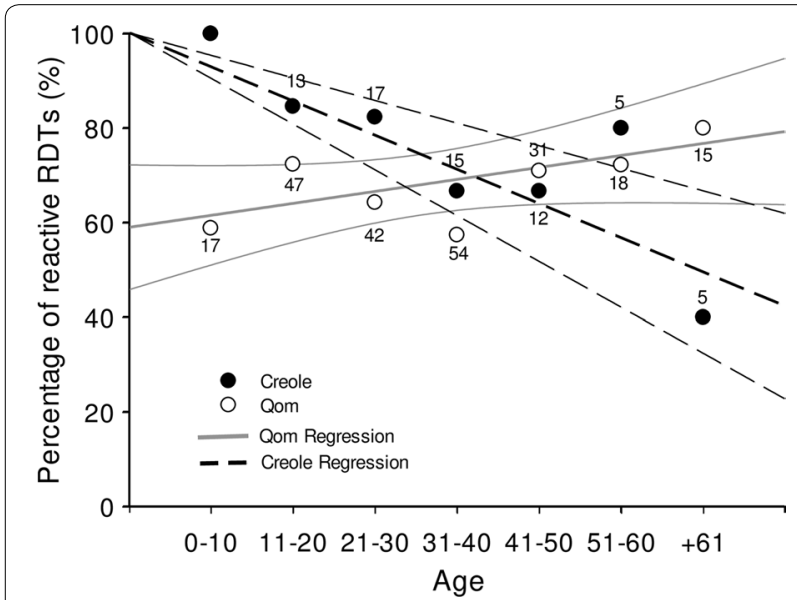

Fig. 3 Chagas Sero K-Set seroreactivity by age and ethnicity, Areas II and IV, Pampa del Indio. Numbers above dots indicate serum samples analysed for each category. Lines represent linear regressions for each ethnicity

Creole communities because of their lower formal education level, tendency not to apply insecticides and lack of screened windows [45]. Creole households have been observed to have a substantially lower risk of triatomine bug and dog infection compared to Qom households in Area I [25].
We also demonstrate that Chagas Sero $K$-SeT is applicable, without modification, to dogs. In two previous studies on $T$. cruzi lineage-specific serology in Argentine dogs $[12,22]$, recombinant TSSA-II/V/VI protein was only used in ELISA. As with humans, we found that there was concordance between ELISA and Chagas Sero $K$-SeT RDT, and that a greater number of T. cruzi seropositive samples tested by both methods were positive with the RDT, confirming the greater sensitivity.

Although we tested a limited number of dogs born after the community-wide insecticide spraying, dogs born prior to this intervention were over eight times more likely to be TSSApep-II/V/VI seropositive, showing the substantially higher risk before the spraying campaign, as well as cumulative risk with age. Qom dog ownership, rather than Creole, was also associated with higher prevalence of TSSApep-II/V/VI seropositivity (Table 2). Furthermore, dogs were more frequently born in Qom communities rather than Creole, which influenced the age that the dogs entered the household, increasing the likelihood of the dog being exposed to triatomines [25]. Owners were asked if the dogs were hunters or guardians and whether the dogs slept inside or outside the domicile; in both of these categories there were not statistically significant differences in Chagas Sero- $K$ SeT result, however, in both cases the categorical divisions may not be entirely definitive.

Trypanosoma cruzi infections in cats are not uncommon, and also occur in domestic mice, which are caught and eaten by cats [46]; however, to our knowledge this is apparently the first application of T. cruzi lineage-specific serology to cats. TSSApep-II/V/VI positive cats had no association with the environmental and behavioural variables listed in Table 2 (data not shown). The Chagas Sero $K$-SeT failed with cats, not unexpectedly; the utility of Protein A, produced naturally by Staphylococcus aureus, rather than Protein G, for binding feline IgG has been reported [47, 48].

Both lineage-specific serology and genotyping indicated the predominance of TcII/V/VI in this endemic region of the Gran Chaco. The Chagas Sero K-SeT RDT demonstrated similar prevalence and clustering in humans and dogs, with ELISAs showing prominent $\mathrm{TcV} /$ VI infections in dogs and cats. Half of the dogs tested here that reacted by ELISA with TSSApep-II/V/VI also reacted with TSSApep-V/VI. TcV and TcVI are the most common genotypes infecting dogs and cats in this area [33].

Genotyping confirmed the association of TcIII with armadillos $[49,50]$. As with the single TcIII infected dog, the Chagas Sero $K$-SeT positivities imply that both that dog and this armadillo were co-infected with TcII, TcV or TcVI. There is clearly a need for more extensive sampling 
among armadillos and sylvatic hosts generally. As with felines, the IgG-binding capacity of Protein A has been exploited in studies on armadilloes (D. novemcinctus), including the use of Protein A-sepharose columns to isolate Ig [51] and of HRP-conjugated Protein A in ELISA to recognise IgG [52].

There is as yet no reliably effective lineage-specific serology for TcI. Reasons for this are unclear, but may be due to the predicted low antigenicity of this isoform of TSSA [17] and perhaps associated with the lack of an ascribed function for TSSA-I, in contrast to TSSA-II/V/ VI [53]. Thus, we cannot exclude some likely co-infections of TcI among the domestic and peridomestic transmission cycles at these study sites. There are relatively low sensitivity ELISAs for TcIII and TcIV [17, 36, 54], and more robust antigens for these lineages would greatly facilitate the study of ecological associations. However, for TcII/V/VI, we have proven here the practicality of deploying lineage-specific serology for surveillance and for enhancing understanding of transmission cycles, and the Chagas Sero $K$-SeT RDT, which is applicable in the field, can give a result in 15 minutes with minimal sample quantities (of whole blood, serum or plasma). Clearly, resolution of the molecular epidemiology of Chagas disease will also continue to benefit from further comparative genomics of $T$. cruzi isolates [55]. Nevertheless, the development of highly sensitive lineage-specific RDTs for all lineages, equally effective for both humans and a wide range of animals, with the aid of Protein G and Protein A detection, would be of great value. This would also allow the enigmatic issue of association of genetic lineage with pathology and prognosis of human Chagas disease to be re-addressed efficiently, and more widely [23].

We acknowledge that the samples used here represent single time-point sampling; however, they provide an antibody profile resultant from both historical and recent $T$. cruzi infections, although that profile may not be comprehensive.

\section{Conclusions}

We have shown that lineage-specific serology can identify T. cruzi infecting lineage, without parasite isolation and genotyping. Furthermore, ELISA is replaceable by an at least equally sensitive RDT, the Chagas Sero $K$-SeT, which incorporates Protein G detection, and is thus directly applicable to humans and several other mammalian species. We assessed lineage distribution among $83 \%$ of the T. cruzi- seropositive human population, showing a statistically significant association of TSSApep-II/V/VI recognition with locality, and with increasing and decreasing age within the Qom and Creole populations, respectively. For dogs TSSApep-II/V/VI seroprevalence was linked to birth before the insecticide spraying programme and with Qom households. The Chagas Sero $K$-SeT is a low cost RDT, applicable for in-the-field surveillance, which can enhance understanding of the transmission pathways and clustering of the lineages, the epidemiology of Chagas disease and the risk of its further emergence from sylvatic cycles. Further research is required to produce corresponding lineage-specific RDTs for T. cruzi lineages TcI, TcIII and TcIV, particularly for TcI.

\section{Supplementary information}

Supplementary information accompanies this paper at https://doi. org/10.1186/s13071-019-3681-7.

Additional file 1: Table S1. Trypanosoma cruzi lineage-specific peptides (TSSApep), with polymorphisms underlined. Table S2. Available corresponding $T$. cruzi genotyping information from humans and animals tested by TSSA lineage-specific serology.

\section{Abbreviations}

ELISA: enzyme-linked immunosorbent assay; GLM: generalized linear model; IFAT: immunofluorescence antibody test; IgG: immunoglobulin G; PCR: polymerase chain reaction; TSSA: trypomastigote small surface antigen; TSSApep: lineage-specific TSSA peptide; RDT: rapid diagnostic test.

\section{Acknowledgements}

We thank Gustavo F. Enriquez, M. Pilar Fernández, Hernán Argibay and M. Marcela Orozco for field and laboratory assistance. We thank Robert H. Gilman for collaboration with the Johns Hopkins Bloomberg School of Public Health, USA. We thank L. Woolfe for photographs of Avia Terai. MVC and REG are members of CONICET Researcher's Career.

\section{Authors' contributions}

NM, NPM and MVC generated the data. NM, NPM, MVC, TB and MAM analysed the data. PM, NZ, YG and QG developed and produced reagents. NM, NPM, MVC, TB, MAM and REG wrote the manuscript. All authors read and approved the final manuscript.

\section{Funding}

Parts of this work were supported by grants from ANPCyT (PICT 20142661), University of Buenos Aires (UBACYT 20020170100779BA and 20020130100843BA), Fundación Bunge \& Born, and the National Institutes of Health, USA (5R01Al107028). The funders had no role in the design of the study, collection, analysis and interpretation of data, or in writing the manuscript. The work undertaken by NM was funded by the Sir Halley Stewart Trust. The views expressed within this article are those of the authors and not necessarily those of the Trust.

\section{Availability of data and materials}

The dataset supporting the conclusions of this article are included within the article and its additional files.

\section{Ethics approval and consent to participate}

Before venepuncture, patients or their parents or guardians provided written informed consent. The procedures for human serological diagnosis and etiological treatment (protocol no. TW-01-004) and the study of parasite diversity have been approved by the "Comité de Etica en Investigación Clínica" (Ethics Committee in Clinical Research) of Buenos Aires, Argentina. All clinical investigations were conducted according to the principles expressed in the Declaration of Helsinki. Animal care and use were performed according to guidelines issued by the Animal Care and Use Committee at the Faculty of Exact and Natural Sciences, University of Buenos Aires, which is based on the International Guiding Principles for Biomedical Research Involving Animals, developed by the Council for International Organizations of Medical Sciences.Archived serum samples that had been obtained during ongoing field work by trained technical staff and stored at the University of Buenos 
Aires were used. Further work and secondary data analysis on these samples was approved by the London School of Hygiene and Tropical Medicine Ethics Committee.

\section{Consent for publication}

Not applicable.

\section{Competing interests}

The authors declare that they have no competing interests.

\section{Author details}

${ }^{1}$ Faculty of Infectious \& Tropical Diseases, London School of Hygiene \& Tropical Medicine, London, UK. ${ }^{2}$ Laboratorio de Eco-Epidemiología, Facultad de Ciencias Exacta y Naturales, Universidad de Buenos Aires, Ciudad Universitaria, C1428EHA Buenos Aires, Argentina. ${ }^{3}$ Consejo Nacional de Investigaciones Científicas y Técnicas-Universidad de Buenos Aires, Instituto de Ecología, Genética y Evolución de Buenos Aires (IEGEBA), Ciudad Universitaria, C1428EHA Buenos Aires, Argentina. ${ }^{4}$ Coris BioConcept, Gembloux, Belgium.

Received: 25 April 2019 Accepted: 22 August 2019

Published online: 16 September 2019

\section{References}

1. Nunes MCP, Beaton A, Acquatella H, Bern C, Bolger AF, Echeverria LE, et al. Chagas cardiomyopathy: An update of current clinical knowledge and management: a scientific statement from the American Heart Association. Circulation. 2018;138:e169-209.

2. Rassi A Jr, Rassi A, Marin-Neto JA. Chagas disease. Lancet. 2010;375:1388-402.

3. World Health Organization. Chagas disease in Latin America: an epidemiological update based on 2010 estimates. Wkly Epidemiol Rec. 2015;90:33-43.

4. Echeverria JE, Bustamante Gomez MB, Pessoa GCDA, Cortez MR, Rodriguez AN, Diotaiuti LG. Resistance to deltamethrin by domestic and wild Triatoma infestans populations in the municipality of Toro Toro, Potosi, Bolivia. Parasites Vectors. 2018;11:92

5. Cardinal MV, Orozco MM, Enriquez GF, Ceballos LA, Gaspe MS, AlvaradoOtegui JA, et al. Heterogeneities in the ecoepidemiology of Trypanosoma cruzi infection in rural communities of the Argentinean Chaco. Am J Trop Med Hyg. 2014:90:1063-73.

6. Orozco MM, Enriquez GF, Cardinal MV, Piccinali RV, Gurtler RE. A comparative study of Trypanosoma cruzi infection in sylvatic mammals from a protected and a disturbed area in the Argentine Chaco. Acta Trop. 2016;155:34-42

7. Zingales B, Andrade SG, Briones MR, Campbell DA, Chiari E, Fernandes O, et al. A new consensus for Trypanosoma cruzi intraspecific nomenclature: second revision meeting recommends Tcl to TcVI. Mem Inst Oswaldo Cruz. 2009;104:1051-4.

8. Lima L, Espinosa-Alvarez O, Ortiz PA, Trejo-Varon JA, Carranza JC, Pinto CM, et al. Genetic diversity of Trypanosoma cruzi in bats, and multilocus phylogenetic and phylogeographical analyses supporting Tcbat as an independent DTU (discrete typing unit). Acta Trop. 2015;151:166-77.

9. Di Noia JM, Buscaglia CA, De Marchi CR, Almeida IC, Frasch AC. A Trypanosoma cruzi small surface molecule provides the first immunological evidence that Chagas' disease is due to a single parasite lineage. J Exp Med. 2002;195:401-13.

10. Bhattacharyya T, Brooks J, Yeo M, Carrasco HJ, Lewis MD, Llewellyn MS, et al. Analysis of molecular diversity of the Trypanosoma cruzi trypomastigote small surface antigen reveals novel epitopes, evidence of positive selection and potential implications for lineage-specific serology. Int J Parasitol. 2010;40:921-8.

11. Burgos JM, Diez M, Vigliano C, Bisio M, Risso M, Duffy T, et al. Molecular identification of Trypanosoma cruzi discrete typing units in end-stage chronic Chagas heart disease and reactivation after heart transplantation. Clin Infect Dis. 2010;51:485-95.

12. Cimino RO, Rumi MM, Ragone P, Lauthier J, D'Amato AA, Quiroga IR, et al. Immuno-enzymatic evaluation of the recombinant TSSA-II protein of
Trypanosoma cruzi in dogs and human sera: a tool for epidemiological studies. Parasitology. 2011;138:995-1002.

13. Risso MG, Sartor PA, Burgos JM, Briceno L, Rodriguez EM, Guhl F, et al. Immunological identification of Trypanosoma cruzi lineages in human infection along the endemic area. Am J Trop Med Hyg. 2011;84:78-84.

14. Bisio M, Seidenstein ME, Burgos JM, Ballering G, Risso M, Pontoriero R, et al. Urbanization of congenital transmission of Trypanosoma cruzi: prospective polymerase chain reaction study in pregnancy. Trans $\mathrm{R}$ Soc Trop Med Hyg. 2011;105:543-9.

15. Vega Benedetti AF, Cimino RO, Cajal PS, Juarez MDV, Villalpando CA, Gil JF, et al. Performance of different Trypanosoma cruzi antigens in the diagnosis of Chagas disease in patients with American cutaneous leishmaniasis from a co-endemic region in Argentina. Trop Med Int Health. 2013;18:1103-9.

16. Longhi SA, Atienza A, Perez Prados G, Buying A, Balouz V, Buscaglia CA, et al. Cytokine production but lack of proliferation in peripheral blood mononuclear cells from chronic Chagas' disease cardiomyopathy patients in response to T. cruzi ribosomal P proteins. PLoS Negl Trop Dis. 2014;8:e2906

17. Bhattacharyya T, Falconar AK, Luquetti AO, Costales JA, Grijalva MJ, Lewis $M D$, et al. Development of peptide-based lineage-specific serology for chronic Chagas disease: geographical and clinical distribution of epitope recognition. PLoS Negl Trop Dis. 2014;8:e2892.

18. Balouz V, Camara Mde L, Canepa GE, Carmona SJ, Volcovich R, Gonzalez $\mathrm{N}$, et al. Mapping antigenic motifs in the trypomastigote small surface antigen from Trypanosoma cruzi. Clin Vaccine Immunol. 2015;22:304-12.

19. Cortina ME, Melli LJ, Roberti M, Mass M, Longinotti G, Tropea S, et al. Electrochemical magnetic microbeads-based biosensor for point-of-care serodiagnosis of infectious diseases. Biosens Bioelectron. 2016;80:24-33.

20. Balouz V, Melli LJ, Volcovich R, Moscatelli G, Moroni S, Gonzalez N, et al. The trypomastigote small surface antigen from Trypanosoma cruzi improves treatment evaluation and diagnosis in pediatric Chagas disease. J Clin Microbiol. 2017:55:3444-53.

21. Peverengo LM, Garcia V, Rodeles LM, Mendicino D, Vicco M, Lagier C, et al. Development and assessment of an improved recombinant multiepitope antigen-based immunoassay to diagnose chronic Chagas disease. Parasitology. 2018;145:1594-9.

22. Floridia-Yapur N, Vega-Benedetti AF, Rumi MM, Ragone P, Lauthier JJ, Tomasini N, et al. Evaluation of recombinant antigens of Trypanosoma cruzi to diagnose infection in naturally infected dogs from Chaco region, Argentina. Parasite Immunol. 2014;36:694-9.

23. Bhattacharyya T, Messenger LA, Bern C, Mertens P, Gilleman Q, Zeippen $\mathrm{N}$, et al. Severity of chagasic cardiomyopathy is associated with response to a novel rapid diagnostic test for Trypanosoma cruzi TcII/N/VI. Clin Infect Dis. 2018;67:519-24.

24. Sartor P, Colaianni I, Cardinal MV, Bua J, Freilij H, Gurtler RE. Improving access to Chagas disease diagnosis and etiologic treatment in remote rural communities of the Argentine Chaco through strengthened primary health care and broad social participation. PLoS Negl Trop Dis. 2017;11:e0005336.

25. Cardinal MV, Sartor PA, Gaspe MS, Enriquez GF, Colaianni I, Gurtler RE. High levels of human infection with Trypanosoma cruzi associated with the domestic density of infected vectors and hosts in a rural area of northeastern Argentina. Parasites Vectors. 2018;11:492.

26. Gurevitz JM, Gaspe MS, Enriquez GF, Vassena CV, Alvarado-Otegui JA Provecho YM, et al. Unexpected failures to control Chagas Disease vectors with pyrethroid spraying in northern Argentina. J Med Entomol. 2012;49:1379-86.

27. Gurevitz JM, Gaspe MS, Enriquez GF, Provecho YM, Kitron U, Gurtler RE. Intensified surveillance and insecticide-based control of the Chagas disease vector Triatoma infestans in the Argentinean Chaco. PLoS Negl Trop Dis. 2013;7:e2158.

28. Gaspe MS, Provecho YM, Fernandez MP, Vassena CV, Santo Orihuela PL, Gurtler RE. Beating the odds: sustained Chagas disease vector control in remote indigenous communities of the Argentine Chaco over a sevenyear period. PLoS Negl Trop Dis. 2018;12:e0006804.

29. Enriquez GF, Cardinal MV, Orozco MM, Schijman AG, Gurtler RE. Detection of Trypanosoma cruzi infection in naturally infected dogs and cats using serological, parasitological and molecular methods. Acta Trop. 2013;126:211-7. 
30. Alvarado-Otegui JA, Ceballos LA, Orozco MM, Enriquez GF, Cardinal MV, Cura C, et al. The sylvatic transmission cycle of Trypanosoma cruzi in a rural area in the humid Chaco of Argentina. Acta Trop. 2012;124:79-86.

31. Orozco MM, Enriquez GF, Alvarado-Otegui JA, Cardinal MV, Schijman AG, Kitron U, et al. New sylvatic hosts of Trypanosoma cruzi and their reservoir competence in the humid Chaco of Argentina: a longitudinal study. Am J Trop Med Hyg. 2013;88:872-82.

32. Burgos JM, Altcheh J, Bisio M, Duffy T, Valadares HM, Seidenstein ME, et al. Direct molecular profiling of minicircle signatures and lineages of Trypanosoma cruzi bloodstream populations causing congenital Chagas disease. Int J Parasitol. 2007;37:1319-27.

33. Enriquez GF, Cardinal MV, Orozco MM, Lanati L, Schijman AG, Gurtler RE. Discrete typing units of Trypanosoma cruzi identified in rural dogs and cats in the humid Argentinean Chaco. Parasitology. 2013;140:303-8.

34. Cosentino RO, Aguero F. A simple strain typing assay for Trypanosoma cruzi: discrimination of major evolutionary lineages from a single amplification product. PLoS Negl Trop Dis. 2012;6:e1777.

35. Macchiaverna NP, Enriquez GF, Buscaglia CA, Balouz V, Gurtler RE, Cardinal MV. New human isolates of Trypanosoma cruzi confirm the predominance of hybrid lineages in domestic transmission cycle of the Argentinean Chaco. Infect Genet Evol. 2018;66:229-35.

36. Kerr CL, Bhattacharyya T, Xavier SC, Barros JH, Lima VS, Jansen AM, Miles MA. Lineage-specific serology confirms Brazilian Atlantic forest lion tamarins, Leontopithecus chrysomelas and Leontopithecus rosalia, as reservoir hosts of Trypanosoma cruzi II (TCII). Parasites Vectors. 2016;9:584.

37. Arenas M, Campos R, Coronado X, Ortiz S, Solari A. Trypanosoma cruzi genotypes of insect vectors and patients with Chagas of Chile studied by means of cytochrome $b$ gene sequencing, minicircle hybridization, and nuclear gene polymorphisms. Vector Borne Zoonotic Dis. 2012;12:196-205.

38. Carrasco HJ, Segovia M, Llewellyn MS, Morocoima A, Urdaneta-Morales S, Martinez C, et al. Geographical distribution of Trypanosoma cruzi genotypes in Venezuela. PLoS Negl Trop Dis. 2012;6:e1707.

39. Cura Cl, Lucero RH, Bisio M, Oshiro E, Formichelli LB, Burgos JM, et al. Trypanosoma cruzi discrete typing units in Chagas disease patients from endemic and non-endemic regions of Argentina. Parasitology. 2012;139:516-21.

40. del Puerto F, Sanchez Z, Nara E, Meza G, Paredes B, Ferreira E, et al. Trypanosoma cruzi lineages detected in congenitally infected infants and Triatoma infestans from the same disease-endemic region under entomologic surveillance in Paraguay. Am J Trop Med Hyg. 2010;82:386-90.

41. del Puerto R, Nishizawa JE, Kikuchi M, lihoshi N, Roca Y, Avilas C, et al. Lineage analysis of circulating Trypanosoma cruzi parasites and their association with clinical forms of Chagas disease in Bolivia. PLoS Negl Trop Dis. 2010;4:e687.

42. Martinez-Perez A, Poveda C, Ramirez JD, Norman F, Girones N, Guhl F, et al. Prevalence of Trypanosoma cruzi's discrete typing units in a cohort of Latin American migrants in Spain. Acta Trop. 2016;157:145-50.

43. Martinez I, Nogueda B, Martinez-Hernandez F, Espinoza B. Microsatellite and mini-exon analysis of Mexican human DTU I Trypanosoma cruzi strains and their susceptibility to nifurtimox and benznidazole. Vector Borne Zoonotic Dis. 2013;13:181-7.

44. Oliveira MT, Branquinho RT, Alessio GD, Mello CGC, Nogueira-de-Paiva NC, Carneiro CM, et al. Tcl, TCll and TcVI Trypanosoma cruzi samples from Chagas disease patients with distinct clinical forms and critical analysis of in vitro and in vivo behavior, response to treatment and infection evolution in murine model. Acta Trop. 2017;167:108-20.

45. Sol Gaspe M, Provecho YM, Cardinal MV, del Pilar Fernandez M, Gurtler RE. Ecological and sociodemographic determinants of house infestation by Triatoma infestans in indigenous communities of the Argentine Chaco. PLoS Negl Trop Dis. 2015;9:e0003614.

46. Gurtler RE, Cardinal MV. Reservoir host competence and the role of domestic and commensal hosts in the transmission of Trypanosoma cruzi. Acta Trop. 2015;151:32-50.

47. Adamczyk B, Tharmalingam-Jaikaran T, Schomberg M, Szekrenyes A, Kelly RM, Karlsson NG, et al. Comparison of separation techniques for the elucidation of IgG N-glycans pooled from healthy mammalian species. Carbohydr Res. 2014;389:174-85.

48. Strietzel CJ, Bergeron LM, Oliphant T, Mutchler VT, Choromanski LJ, Bainbridge G. In vitro functional characterization of feline lgGs. Vet Immunol Immunopathol. 2014;158:214-23.

49. Yeo M, Acosta N, Llewellyn M, Sanchez H, Adamson S, Miles GA, et al. Origins of Chagas disease: Didelphis species are natural hosts of Trypanosoma cruzi I and armadillos hosts of Trypanosoma cruzi ll, including hybrids. Int J Parasitol. 2005;35:225-33.

50. Llewellyn MS, Lewis MD, Acosta N, Yeo M, Carrasco HJ, Segovia M, et al. Trypanosoma cruzi llc: phylogenetic and phylogeographic insights from sequence and microsatellite analysis and potential impact on emergent Chagas disease. PLoS Negl Trop Dis. 2009;3:e510.

51. Fernandes GF, Deps P, Tomimori-Yamashita J, Camargo ZP. IgM and IgG antibody response to Paracoccidioides brasiliensis in naturally infected wild armadillos (Dasypus novemcinctus). Med Mycol. 2004;42:363-8.

52. Duthie MS, Truman RW, Goto W, O'Donnell J, Hay MN, Spencer JS, et al. Insight toward early diagnosis of leprosy through analysis of the developing antibody responses of Mycobacterium leprae-infected armadillos. Clin Vaccine Immunol. 2011;18:254-9.

53. Camara MLM, Canepa GE, Lantos AB, BalouzV, Yu H, Chen $X$, et al. The trypomastigote small surface antigen (TSSA) regulates Trypanosoma cruzi infectivity and differentiation. PLoS NegI Trop Dis. 2017;11:e0005856.

54. Bhattacharyya T, Mills EA, Jansen AM, Miles MA. Prospects for T. cruzi lineage-specific serological surveillance of wild mammals. Acta Trop. 2015;151:182-6.

55. Messenger LA, Miles MA, Bern C. Between a bug and a hard place: Trypanosoma cruzi genetic diversity and the clinical outcomes of Chagas disease. Expert Rev Anti Infect Ther. 2015;13:995-1029.

\section{Publisher's Note}

Springer Nature remains neutral with regard to jurisdictional claims in published maps and institutional affiliations.

\footnotetext{
Ready to submit your research? Choose BMC and benefit from:

- fast, convenient online submission

- thorough peer review by experienced researchers in your field

- rapid publication on acceptance

- support for research data, including large and complex data types

- gold Open Access which fosters wider collaboration and increased citations

- maximum visibility for your research: over $100 \mathrm{M}$ website views per year
}

At BMC, research is always in progress.

Learn more biomedcentral.com/submissions 